\title{
CT assessment of the quality of omentoplasty and its implications for perineal wound healing
}

\author{
Robin D. Blok ${ }^{1,2}$ (D) Sarah Sharabiany ${ }^{1} \cdot$ Colin G. Ferrett $^{3} \cdot$ Roel Hompes $^{1} \cdot$ Pieter J. Tanis ${ }^{1} \cdot$ Christopher Cunningham $^{4}$
}

Accepted: 23 September 2019 / Published online: 4 November 2019

(C) The Author(s) 2019

\begin{abstract}
Purpose This study was designed to examine the impact of an omentoplasty and its quality on pelviperineal morbidity after abdominoperineal resection (APR) for rectal cancer.

Methods This was a retrospective single-centre study of consecutive patients undergoing APR for primary or recurrent rectal cancer between 2000 and 2018. Quality of omentoplasty was categorised (sufficient vs insufficient) based on postoperative CT scans. Main study endpoints were perineal wound healing and perineal hernia.

Results This study included 100 patients: 16 with a sufficient omentoplasty, 16 with an insufficient omentoplasty, and 68 without omentoplasty. Rate of pelviperineal complications within 30 days was $44 \%, 69 \%$ and $64 \%(P=0.283)$, and delayed wound healing at 3 months was $19 \%, 54 \%$ and $27 \%$, respectively $(P=0.109)$. Sufficient omentoplasty was not significantly associated with less delayed healing in multivariable analysis (OR 0.597; 95\% CI $0.149-$ 2.397). An insufficient omentoplasty demonstrated significantly higher rates of delayed healing at 6 months (46\% vs $14 \% ; P=0.016)$ and chronic perineal sinus at 12 months $(31 \%$ vs $3 \% ; P=0.008)$ compared with no omentoplasty.

Conclusion This relatively small series suggest that even a sufficient omentoplasty, as determined by postoperative imaging, does not reduce pelviperineal morbidity after APR for rectal cancer. The methodology of CT-based assessment of an omentoplasty as well as the correlation with postoperative outcomes has to be validated in future studies.
\end{abstract}

Keywords Rectal neoplasms $\cdot$ Abdominoperineal resection $\cdot$ Omentoplasty $\cdot$ Perineal wound healing $\cdot$ Perineal herniation

Electronic supplementary material The online version of this article (https://doi.org/10.1007/s00384-019-03412-0) contains supplementary material, which is available to authorized users.

Pieter J. Tanis

P.J.Tanis@amsterdamumc.nl

1 Department of Surgery, Amsterdam University Medical Centres, University of Amsterdam, Post box 22660, 1100 DD Amsterdam, The Netherlands

2 LEXOR, Centre for Experimental and Molecular Medicine, Oncode Institute, Cancer Centre Amsterdam, Amsterdam UMC, University of Amsterdam, Amsterdam, The Netherlands

3 Department of Radiology, Oxford University Hospitals NHS Foundation Trust, Oxford, UK

4 Department of Colorectal Surgery, Churchill Hospital, Oxford University Hospitals NHS Foundation Trust, Oxford, UK

\section{Introduction}

The treatment for distal rectal cancer may require an abdominoperineal resection (APR). The creation of a dead space at the site of the excised rectum and anal sphincter complex after APR commonly results in perineal infection, breakdown and delayed wound healing $[1,2]$. Patients having preoperative radiotherapy and requiring a wider excision due to locally advanced disease are at increased risk of suffering from perineal wound complications [3-5].

Omentoplasty (OP) has been advocated to reduce the frequency of perineal wound problems and prevent the small bowel from entering into the narrow pelvis with a risk of getting trapped [6]. However, application of OP is currently highly debated as there is increasing evidence to suggest that OP does not affect the occurrence of pelviperineal wound complications after APR for rectal cancer [7, 8]. Moreover, there seems a similar need for operative management of small 
bowel obstruction and even an increased risk of perineal herniation in patients who have had OP [9].

Inefficacy of OP might be related to quality as defined by insufficient length or insufficient bulk to ensure adequate obliteration of the pelvic resection space. But this hypothesis has never been tested. The best method for assessing the quality of surgery seems by means of postoperative radiological evaluation, rather than intraoperative judgement of the surgeon [10].

The purpose of this study therefore was to check the sufficiency of OP based on postoperative radiological imaging and to evaluate its implications for pelviperineal wound complications and related morbidity.

\section{Method}

\section{Patients and design}

All APR patients for primary or recurrent rectal cancer at a single institution between 2010 and 2018 were identified. Exclusion criteria were other underlying disease, total pelvic exenteration, perineal closure using a muscle flap (because of the inability to determine the quality of pelvic filling by OP) and no evaluable radiological imaging post APR.

Patient data were extracted via electronic and handwritten medical records and included baseline characteristics, operative details, early and late surgical complications and related readmission and reintervention. To ensure sufficient follow-up, all complications were recorded for 18 months after surgery, except for perineal hernia status and related reoperation, which were recorded for 5 years after surgery. Patients were divided into three groups (sufficient $\mathrm{OP}$, insufficient $\mathrm{OP}$, and no OP), based on the operative reports and assessment of the postoperative $\mathrm{CT}$ scans.

The study was sponsored by the Oxford University Hospitals NHS Foundation Trust, and received approval of the local Institutional Review Board (Cambridge East Research Ethics Committee). Patients were studied as part of a retrospective surgical audit and written informed consent was not required. The study was reported according to the Strengthening the Reporting of Observational studies in Epidemiology (STROBE) guidelines [11].

\section{Outcome measures}

The primary endpoint was delayed wound healing at 3 months after surgery, defined as any complicated perineal wound healing present beyond 3 months. Secondary endpoints were percentage of patients with one or more pelviperineal complications within 30 days, rate of delayed healing at 6 months, chronic perineal sinus at 1 year, overall and specific pelviperineal complications until end of follow-up, perineal herniation (clinical diagnosis with radiological confirmation), small bowel obstruction at the level of the pelvis (not based on recurrent cancer), OP-related complications, and overall and specific need for related readmission and reintervention (including medical, radiological and surgical interventions).

\section{Radiological assessment}

Routine oncological follow-up included a pelvic CT scan 1 year after APR. These scans were reviewed for the quality of the OP and the presence of a perineal hernia as well as its content. The quality of the OP was recorded as sufficient when the omental pedicle reached fully onto the pelvic wound bed (i.e. sufficient length) and provided sufficient volume to fully fill the pelvic cavity with absence of small bowel in the lower pelvis (Fig. 1a). The quality was recorded as insufficient when there was either insufficient length (Fig. 1b) or insufficient volume with interposition of small bowel in the pelvis (Fig. 1c). Radiologically, perineal herniation was defined as visceral descent below the perineal body line (Fig. 2) [12]. All scans were reviewed by a specialist radiologist (CGF) and one of two authors (RDB or SS). Any disagreement was resolved through consensus discussion with a fourth author (PJT).

\section{Statistics}

Categorical data were compared using the Fischer's exact test, and numerical data with the one-way ANOVA and KruskalWallis test, according to distribution. Multiple logistic regression with stepwise backward selection was used to account for potential confounding. Covariates were selected on a theoretical basis with a maximum of independent variables that the data allowed for. The Kaplan-Meier method was used to describe small bowel obstruction according to the use and quality of OP, and Cox regression was used to relate several predictors to the development of perineal hernia over time. Cases with missing values were not included in the analyses. The significance threshold was set at $P$ value $<0.05$. All analyses were performed using IBM SPSS statistics, version 25.0.0 (IBM Corporation, Armonk, NY, USA).

\section{Results}

\section{Patient characteristics}

Between January 2010 and July 2018, a total of 119 consecutive patients underwent APR at the Oxford University Hospitals. After exclusion of duplicate records $(n=1)$, other underlying disease $(n=2)$, total pelvic exenteration $(n=8)$ and muscle flap closure $(n=8), 100$ patients were included in the analyses. Median time to pelvic imaging in patients who underwent OP was 11 months postoperatively (IQR 7-12). Radiological assessment qualified 16 patients as having a 


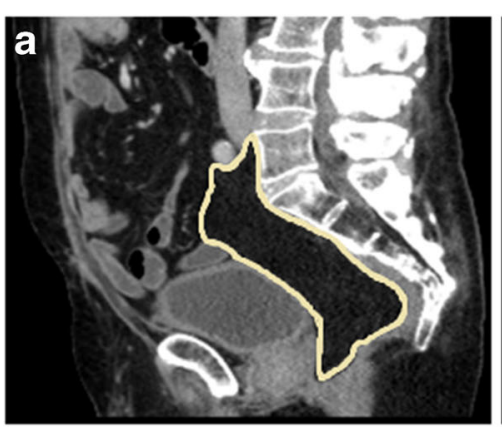

Fig. 1 Mid-sagittal CT images of the pelvis of three male patients 1 year after abdominoperineal resection for rectal cancer. a The omentoplasty has sufficient length and volume to fill the pelvic resection space, and supports the bladder and prostate without interposition of small bowel. b

sufficient $\mathrm{OP}$ and 16 as having an insufficient $\mathrm{OP}$, and the remaining 68 patients did not undergo OP.

Baseline characteristics are demonstrated in Table 1, and stratified by presence and quality of OP. Mean age of the cohort was 66 years $( \pm 13)$. The included patients were predominantly male (70\%). Preoperative radiotherapy was given in 81 to $87 \%(P=$ $0.907)$ and adjuvant chemotherapy in 31 to $43 \%(P=0.800)$ among the three patient groups. A history of hysterectomy was more common in the insufficient OP group $(P=0.025)$. Surgical details are described in Table 2 . There were no substantial differences, except for fewer coccygectomy $(P=0.133)$ and posterior vaginectomy $(P=0.088)$ in patients without OP. The amount of levator resection and method of perineal wound closure were well balanced between the three groups (Table 2).

\section{Pelviperineal wound healing}

Length of in-hospital stay was not significantly different between the three groups $(P=0.083)$ (Supplementary Table 1$)$. Pelviperineal wound complications within 30 days occurred in 7/16 patients (44\%) following APR with sufficient OP. Corresponding numbers were $9 / 13$ patients (69\%) and 40/63 patients $(64 \%)$ following an insufficient OP and no OP,
The omentoplasty has insufficient length to reach onto the pelvic wound bed. $\mathbf{c}$ The omentoplasty has adequate length, but insufficient volume to fill the pelvic resection space with clear interposition of small bowel in the pelvis

respectively $(P=0.283)$ (Supplementary Table 2). Multiple logistic regression with stepwise backward selection did not reveal any significant association between 30-day complications and use of OP $(P=0.294)$, nor with preoperative radiotherapy, extralevator resection or adjacent organ resection (Table 3).

Delayed wound healing at 3 months was not significantly different between patients with a sufficient OP (3/16 (19\%)), an insufficient OP (7/13 (54\%)) and no OP (18/66 (27\%)) $(P=$ 0.109). Multiple logistic regression confirmed the absence of significant associations between the use of $\mathrm{OP}$ and delayed wound healing at 3 months $(P=0.092)$ (Table 3$)$. Extralevator APR was the only independent predictor of delayed healing in the model (OR 5.275; 95\% CI 1.050-26.501; $P=0.043$ ).

Over time, perineal wound healing was achieved significantly less often in patients that showed an insufficient OP on postoperative radiological imaging (Supplementary Table 2). Delayed healing at 6 months was present in $6 / 13$ patients (46\%; $P=0.004)$, and $4 / 13$ patients $(31 \% ; P=0.007)$ still had a chronic perineal sinus at 12 months. Reoperation for a perineal wound problem was also more frequent in the insufficient OP group $(P=0.038)$ (Supplementary Table 3$)$. Comparison of sufficient OP and no OP showed no statistically significant differences in the rates of delayed wound
Fig. 2 Mid-sagittal CT images of two patients 1 year after abdominoperineal resection. a Male patient with omentoplasty in anatomical position and no herniation below the line drawn between the perineal body (arrow) and the coccyx. b Female patient with a large omental hernia
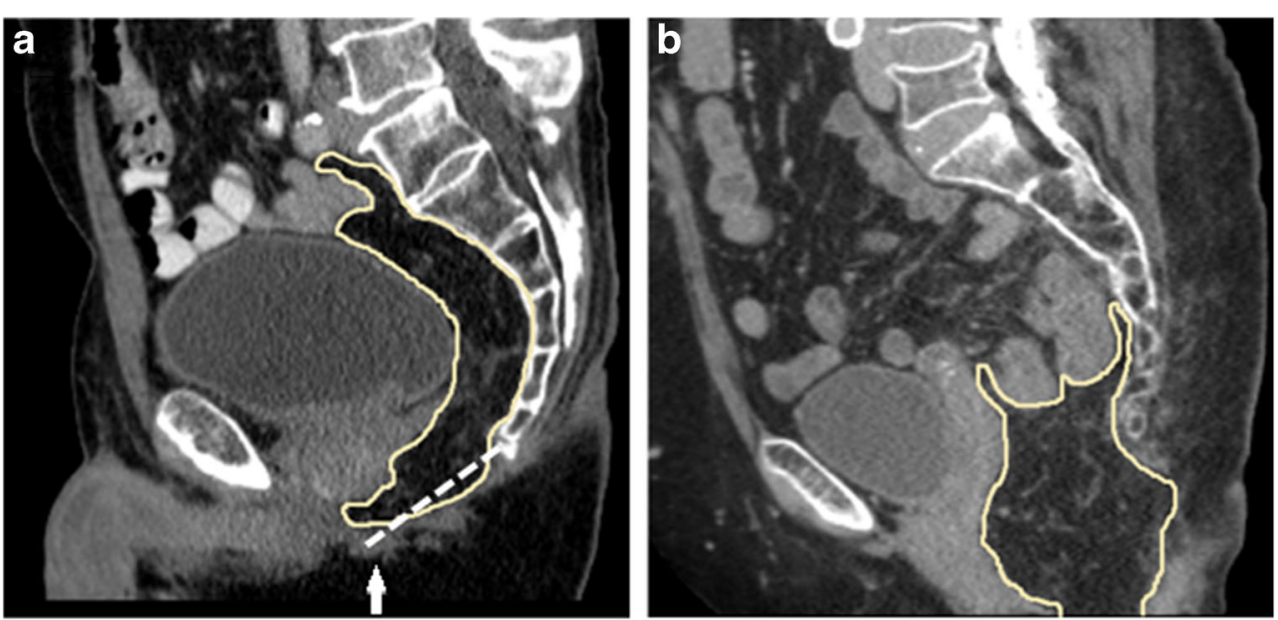
Table 1 Baseline characteristics

\begin{tabular}{|c|c|c|c|c|c|}
\hline Parameters & & $\begin{array}{l}\text { Sufficient } \\
\text { omentoplasty } \\
(N=16)\end{array}$ & $\begin{array}{l}\text { Insufficient } \\
\text { omentoplasty } \\
(N=16)\end{array}$ & No omentoplasty $(N=68)$ & $P$ value \\
\hline Gender & Male & $12 / 16(75)$ & $8 / 16(50)$ & $50 / 68(74)$ & 0.183 \\
\hline \multirow[t]{2}{*}{ Age } & Years (mean $\pm \mathrm{SD}$ ) & $67 \pm 11$ & $66 \pm 7$ & $65 \pm 15$ & 0.894 \\
\hline & $>65$ years & $9 / 16(56)$ & $11 / 16(69)$ & $39 / 68(57)$ & 0.751 \\
\hline BMI & $\mathrm{kg} / \mathrm{m}^{2}($ mean $\pm \mathrm{SD})$ & $26 \pm 3$ & $29 \pm 6$ & $28 \pm 5$ & 0.468 \\
\hline \multirow[t]{3}{*}{ ASA-classification $^{\mathrm{a}}$} & ASA I & 2/16 (13) & $1 / 15(7)$ & $5 / 67(8)$ & 0.848 \\
\hline & ASA II & $12 / 16(75)$ & $10 / 15(67)$ & $47 / 67(70)$ & 0.893 \\
\hline & ASA III & $2 / 16(13)$ & $4 / 15(27)$ & $15 / 67(22)$ & 0.606 \\
\hline \multirow[t]{2}{*}{ Smoking status } & Active smoker & 3/16 (19) & $3 / 15(20)$ & $7 / 64(11)$ & 0.431 \\
\hline & Cessation smoking $<10$ years & $0 / 16(0)$ & $3 / 15(20)$ & $11 / 64(17)$ & 0.169 \\
\hline \multirow[t]{2}{*}{ Comorbidities } & Diabetes mellitus & $1 / 16(6)$ & 3/16 (19) & $8 / 67(12)$ & 0.652 \\
\hline & Vascular disease & $3 / 16(19)$ & $4 / 15(27)$ & $11 / 66(17)$ & 0.620 \\
\hline Prior abdominal surgery & Total & $5 / 16(31)$ & $10 / 15(67)$ & $28 / 68(41)$ & 0.111 \\
\hline \multirow[t]{2}{*}{ Prior pelvic surgery } & Total & $5 / 16(31)$ & $8 / 15(53)$ & $19 / 68(28)$ & 0.170 \\
\hline & Hysterectomy & $0 / 16(0)$ & 4/16 (25) & $3 / 68(4)$ & 0.025 \\
\hline \multirow[t]{2}{*}{ Primary disease } & Primary rectal cancer & $13 / 16(81)$ & $12 / 16(75)$ & $57 / 68(84)$ & 0.626 \\
\hline & Recurrent rectal cancer & $3 / 16(19)$ & $4 / 16(25)$ & $11 / 68(16)$ & 0.626 \\
\hline \multirow[t]{4}{*}{ Clinical tumour stage } & Stage I & $1 / 16(6)$ & 4/12 (33) & $16 / 58(28)$ & 0.140 \\
\hline & Stage II & $4 / 16(25)$ & $2 / 12(17)$ & $20 / 58(35)$ & 0.464 \\
\hline & Stage III & $10 / 16(63)$ & $5 / 12(42)$ & $20 / 58(35)$ & 0.145 \\
\hline & Stage IV & $1 / 16(6)$ & $1 / 12(8)$ & $2 / 58(3)$ & 0.393 \\
\hline \multirow[t]{4}{*}{ Neoadjuvant therapy } & None & 2/16 (13) & 3/16 (19) & $9 / 66(14)$ & 0.907 \\
\hline & Short-course radiotherapy & $1 / 16(6)$ & $0 / 16(0)$ & $2 / 66(3)$ & 0.699 \\
\hline & Long-course radiotherapy & $0 / 16(0)$ & $1 / 16(6)$ & $5 / 66(8)$ & 0.825 \\
\hline & Chemo-radiotherapy & $13 / 16(81)$ & $12 / 16(75)$ & $50 / 66(76)$ & 0.940 \\
\hline \multirow[t]{5}{*}{ Pathological tumour stage } & Complete response & $1 / 16(6)$ & $2 / 16(13)$ & $8 / 67(12)$ & 1.000 \\
\hline & Stage I & $5 / 16(31)$ & $6 / 16(38)$ & 13/67 (19) & 0.252 \\
\hline & Stage II & $6 / 16(38)$ & $6 / 16(38)$ & 23/67 (34) & 1.000 \\
\hline & Stage III & $3 / 16(19)$ & 2/16 (13) & $22 / 67(33)$ & 0.209 \\
\hline & Stage IV & $1 / 16(6)$ & $0 / 16(0)$ & $1 / 67(2)$ & 0.544 \\
\hline Adjuvant chemotherapy & Total & $5 / 16(31)$ & $6 / 14(43)$ & $22 / 67(33)$ & 0.800 \\
\hline Follow-up duration & Months (median + IQR) & $18(17-20)$ & $19(17-22)$ & $17(13-18)$ & 0.033 \\
\hline
\end{tabular}

${ }^{a}$ American Society of Anaesthesiologists (ASA) physical status classification system

healing at 6 months $(0 / 15(0 \%)$ vs $9 / 64(14 \%) ; P=0.195)$ and chronic perineal sinus formation at 12 months $(0 / 14(0 \%)$ vs $2 / 59(3 \%) ; P=1.000)$, respectively. The number of events was too small to allow adjustment in a multivariable model.

Overall presence of one or more pelviperineal complications within 1 year was recorded in $9 / 16$ patients $(56 \%)$ with a sufficient OP, in 10/15 patients (67\%) with an insufficient OP, and in 46/66 patients $(70 \%)$ without $\mathrm{OP}(P=0.610)$. Again, the association of $\mathrm{OP}$ and pelviperineal complications within 1 year remained non-significant after adjustment for potential confounding $(P=0.595)$ (Table 3$)$. Nor were there any significant associations between the three groups and occurrence of specific perineal wound-related problems (Supplementary Table 2).

\section{Perineal herniation}

The median follow-up duration for assessing perineal hernia status was 33 months (IQR 16-51). A clinical diagnosis of perineal hernia was significantly more common after use of OP, with $4 / 16$ hernias (25\%) in those with a sufficient OP and $5 / 15$ hernias $(33 \%)$ in those with an insufficient OP, compared with $7 / 68$ hernias $(10 \%)$ in those without OP $(P=0.036)$ (Supplementary Table 4). Patients with a sufficient OP showed a trend to have more frequent surgical correction of a perineal hernia (13\% following sufficient OP vs $7 \%$ following insufficient $\mathrm{OP}$ and $2 \%$ following no OP; $P=0.090$ ) (Supplementary Table 3). The hazard of perineal hernia formation over time, stratified by group, and adjusted for 
Table 2 Operative procedures

\begin{tabular}{|c|c|c|c|c|c|}
\hline Parameters & & $\begin{array}{l}\text { Sufficient omentoplasty } \\
(N=16)\end{array}$ & $\begin{array}{l}\text { Insufficient omentoplasty } \\
(N=16)\end{array}$ & $\begin{array}{l}\text { No omentoplasty } \\
(N=68)\end{array}$ & $P$ value \\
\hline \multirow[t]{3}{*}{ Type of surgery } & Intersphincteric $\mathrm{APR}^{\mathrm{a}}$ & 2/16 (13) & $0 / 14(0)$ & $7 / 68(10)$ & 0.569 \\
\hline & Conventional $\mathrm{APR}^{\mathrm{a}}$ & $1 / 16(6)$ & $3 / 14(21)$ & $7 / 68(10)$ & 0.479 \\
\hline & Extralevator $\mathrm{APR}^{\mathrm{a}}$ & $13 / 16(81)$ & $11 / 14(79)$ & $54 / 68(79)$ & 1.000 \\
\hline \multirow[t]{3}{*}{ Abdominal approach } & Open & $3 / 16(19)$ & $7 / 16(44)$ & 13/68 (19) & 0.129 \\
\hline & Laparoscopic & $13 / 16(81)$ & $9 / 16(56)$ & $55 / 68(81)$ & 0.129 \\
\hline & Conversion $^{\mathrm{b}}$ & $0 / 13(0)$ & $3 / 9(33)$ & $7 / 55(13)$ & 0.056 \\
\hline \multirow[t]{3}{*}{ Perineal approach } & Open & $15 / 16(94)$ & $12 / 13(92)$ & $46 / 68(68)$ & 0.028 \\
\hline & Transperineal minimally invasive & $1 / 16(6)$ & $1 / 13(8)$ & $22 / 68(32)$ & 0.028 \\
\hline & Conversion $^{c}$ & $0 / 1(0)$ & $0 / 1(0)$ & $1 / 22(5)$ & 1.000 \\
\hline \multirow[t]{2}{*}{ Surgical position } & Prone & $10 / 16(63)$ & $5 / 16(31)$ & $26 / 68(38)$ & 0.170 \\
\hline & Lithotomy & $6 / 16(38)$ & $11 / 16(69)$ & $42 / 68(62)$ & 0.170 \\
\hline \multirow[t]{7}{*}{ Adjacent organ resection } & Total & $12 / 16(75)$ & $9 / 14(64)$ & $27 / 68(40)$ & 0.021 \\
\hline & Coccyx & $8 / 16(50)$ & $6 / 14(43)$ & $18 / 68(27)$ & 0.133 \\
\hline & Partial prostate and/or vesicula & 2/16 (13) & $0 / 14(0)$ & $5 / 68(7)$ & 0.495 \\
\hline & Vaginal wall & 2/16 (13) & $3 / 14(21)$ & $4 / 68(6)$ & 0.088 \\
\hline & Uterus & $0 / 16(0)$ & $0 / 14(0)$ & $2 / 68(3)$ & 1.000 \\
\hline & Pelvic sidewall & $1 / 16(6)$ & $0 / 14(0)$ & $1 / 68(2)$ & 0.521 \\
\hline & Urinary tract & $1 / 16(6)$ & $0 / 14(0)$ & $2 / 68(3)$ & 0.670 \\
\hline \multirow[t]{2}{*}{ Visceral interposition } & Uterus & $0 / 16(0)$ & $1 / 13(8)$ & $1 / 67(2)$ & 0.280 \\
\hline & Bladder & $0 / 16(0)$ & $1 / 13(8)$ & $0 / 67(0)$ & 0.135 \\
\hline \multirow[t]{3}{*}{ Perineal closure } & Primarily & $7 / 16(44)$ & $6 / 12(50)$ & $31 / 68(46)$ & 1.000 \\
\hline & Biomesh & $1 / 16(6)$ & $1 / 12(8)$ & $7 / 68(10)$ & 1.000 \\
\hline & Vicryl mesh & $8 / 16(50)$ & $5 / 12(42)$ & $29 / 68(43)$ & 0.899 \\
\hline Pelvic drain & Total & $13 / 16(81)$ & $9 / 13(69)$ & $53 / 68(78)$ & 0.747 \\
\hline Perineal drain & Total & $12 / 16(75)$ & $7 / 13(54)$ & $40 / 68(59)$ & 0.403 \\
\hline Prolonged antibiotic continuation & Total & $4 / 16(25)$ & $6 / 14(43)$ & $27 / 68(40)$ & 0.532 \\
\hline V.A.C. ${ }^{\mathrm{d}}$ placement & Total & $2 / 16(13)$ & $0 / 13(0)$ & $2 / 68(3)$ & 0.209 \\
\hline Intra-operative complication & Total & 2/16 (13) & $3 / 14(21)$ & $13 / 68(19)$ & 0.850 \\
\hline
\end{tabular}

${ }^{\mathrm{a}} A P R$ abdominoperineal resection

${ }^{\mathrm{b}}$ Percentage from laparoscopic group

${ }^{\mathrm{c}}$ Percentage from transperineal minimally invasive group

${ }^{\mathrm{d}}$ Vacuum-assisted closure

hysterectomy and resection of the coccyx, is illustrated in Fig. 3. The Cox proportional hazards model revealed that the use of OP did not significantly influence the hazard of perineal hernia formation. Hysterectomy was associated with an increased rate of perineal hernia (HR 7.796; 95\% CI 2.318-26.221) (Table 4). The contents of the hernia sac among 15 patients in whom a perineal hernia was detected at routine follow-up CT scans revealed omentum in $5 / 5$ patients in the sufficient OP group, and small bowel in 3/3 patients in the insufficient OP group and $5 / 7$ patients in the no OP group (Supplementary Table 4).

\section{Small bowel obstruction}

Overall, $10 \%$ of patients demonstrated small bowel obstruction at the level of the pelvic cavity. Reported causes for obstruction were adhesions $(n=4)$ and obstruction in an infiltrative necrotic area $(n=1)$. In five cases, dilated loops of small bowel with a transition point in the pelvis were seen without clear cause. The cumulative incidences of pelvic ileus were $0 \%$ (sufficient OP), 7\% (insufficient OP) and 14\% (no OP). The occurrence of small bowel obstruction over time did not significantly differ between the three groups $(P=0.246)$ (Fig. 4), nor did the need for reoperation for this problem $(P=$ 1.000) (Supplementary Table 3).

\section{Omental flap complications}

One out of $32(3 \%)$ constructions of OP was found to be complicated postoperatively, which was discovered during laparoscopy for debridement of a pelvic abscess cavity 1 
Table 3 Multiple logistic regression analyses with stepwise backward selection

\begin{tabular}{|c|c|c|c|c|c|c|}
\hline \multirow[t]{2}{*}{ Parameters } & \multicolumn{2}{|c|}{ Wound problem within 30 days p.o. } & \multicolumn{2}{|c|}{ Unhealed perineal wound at 3 months p.o. } & \multicolumn{2}{|c|}{ Wound problem within 1 year p.o. } \\
\hline & OR $(95 \% \mathrm{CI})$ & $P$ value & OR $(95 \% \mathrm{CI})$ & $P$ value & OR $(95 \% \mathrm{CI})$ & $P$ value \\
\hline \multicolumn{7}{|l|}{ Unadjusted } \\
\hline Omentoplasty $^{\mathrm{a}}$ & & 0.294 & & 0.110 & & 0.595 \\
\hline Sufficient omentoplasty & $0.447(0.147-1.361)$ & & $0.615(0.157-2.415)$ & & $0.559(0.183-1.711)$ & \\
\hline Insufficient omentoplasty & $1.294(0.358-4.675)$ & & $3.111(0.921-10.512)$ & & $0.870(0.263-2.873)$ & \\
\hline \multicolumn{7}{|l|}{ Adjusted } \\
\hline Omentoplasty $^{\mathrm{a}}$ & & $-{ }^{\mathrm{c}}$ & & 0.092 & & $--^{\mathrm{c}}$ \\
\hline Sufficient omentoplasty & & $-{ }^{\mathrm{c}}$ & $0.597(0.149-2.397)$ & & & $-{ }^{\mathrm{c}}$ \\
\hline Insufficient omentoplasty & & $-^{\mathrm{c}}$ & $3.985(0.976-16.283)$ & & & $-^{\mathrm{c}}$ \\
\hline Any radiotherapy & & $\mathrm{ns}^{\mathrm{d}}$ & & $\mathrm{ns}^{\mathrm{d}}$ & & $\mathrm{ns}^{\mathrm{d}}$ \\
\hline Extralevator $\mathrm{APR}^{\mathrm{b}}$ & & $\mathrm{ns}^{\mathrm{d}}$ & $5.275(1.050-26.501)$ & 0.043 & & $\mathrm{~ns}^{\mathrm{d}}$ \\
\hline Adjacent organ resection & & $\mathrm{ns}^{\mathrm{d}}$ & & $\mathrm{ns}^{\mathrm{d}}$ & & $n \mathrm{~s}^{\mathrm{d}}$ \\
\hline
\end{tabular}

${ }^{a}$ Entered as categorical variable including sufficient omentoplasty, insufficient omentoplasty or no omentoplasty

${ }^{\mathrm{b}} A P R$ abdominoperineal resection

${ }^{\mathrm{c}}$ There were no significant predictors retained in the model

${ }^{\mathrm{d}}$ ns non-significant

month after APR. A huge cavity was seen with necrotic omentum posterior to the wound site, likely to be responsible for the perineal infection. A second patient demonstrated an omental defect of $1.5 \mathrm{~cm}$ with small bowel passing through, which was discovered during surgical drainage of a perineal abscess, but no obstruction was present at the time.

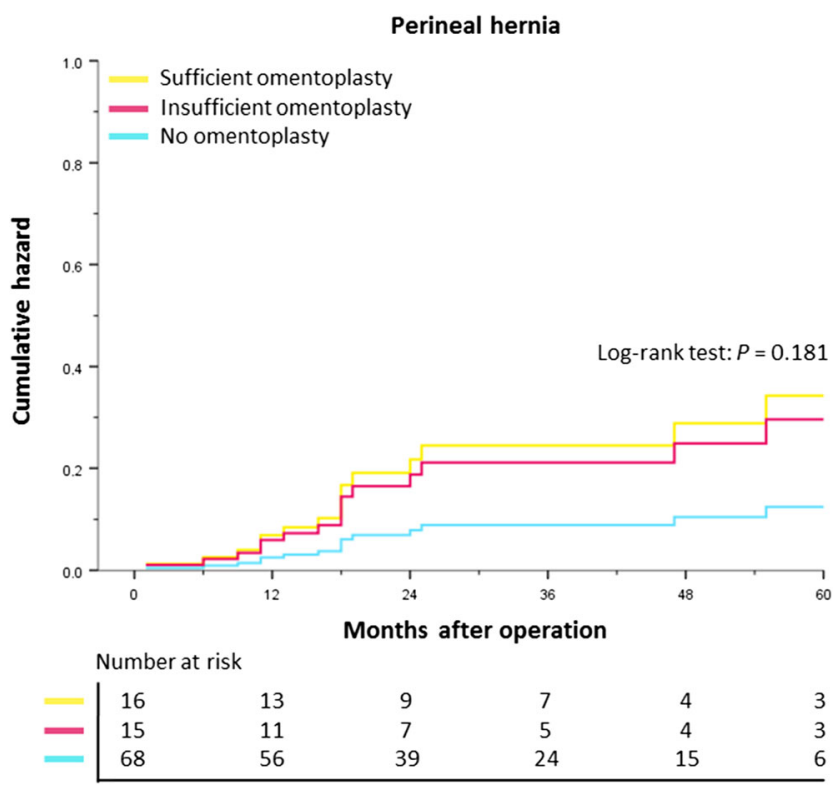

Fig. 3 Adjusted Cox regression survival curve for clinical diagnosis of perineal hernia after abdominoperineal resection, stratified by the use and quality of omentoplasty. Lines are corrected for hysterectomy and coccygectomy

\section{Discussion}

The current results suggest that even a sufficient OP, as determined on postoperative radiological imaging, does not confer any additional benefits in terms of perineal wound healing after APR for rectal cancer, although numbers are small. Reoperation for small bowel obstruction was also not significantly reduced after OP. The OP itself showed a trend to increasing the risk of perineal herniation, with the OP being the content of the hernia sac in all five patients with perineal hernia and a sufficient OP.

Similar perineal infection and wound healing rates were observed after an OP of sufficient length and volume in comparison with no OP. One might therefore conclude that the type of tissue used to fill the pelvic dead space after APR is unimportant. In the absence of OP, small bowel might function as an excellent presacral padding for a primary clean-contaminated APR for

Table 4 Cox proportional hazards model

\begin{tabular}{llc}
\hline Parameters & \multicolumn{2}{l}{ Clinically diagnosed perineal hernia } \\
\cline { 2 - 3 } & Hazard ratio (CI 95\%) & $P$ value \\
\hline Omentoplasty $^{\mathrm{a}}$ & & 0.181 \\
Sufficient omentoplasty $^{\mathrm{b}}$ & $2.749(0.739-10.225)$ & \\
Insufficient omentoplasty $^{\mathrm{b}}$ & $2.374(0.694-8.122)$ & \\
Hysterectomy $_{\text {Coccygectomy }}$ & $7.796(2.318-26.221)$ & 0.001 \\
& $2.787(0.964-8.057)$ & 0.058
\end{tabular}

${ }^{a}$ Entered as categorical variable including sufficient omentoplasty, insufficient omentoplasty or no omentoplasty

${ }^{\mathrm{b}}$ Reference: no omentoplasty 
rectal cancer, as there seems no increase in problems such as abscesses or small bowel fistulas. This may also indicate that perhaps the main problem in primary APR does not arise from the presacral cavity, but from the resected space of the anal sphincter complex. The OP predominantly fills the presacral space and does little to prevent dead space formation in the excised anal canal. This could explain the fact that despite sufficient length and volume, an OP was not superior to primary wound closure in this study. It therefore may be important to distinguish primary APR from a situation with a clear infectious problem located in the pelvis $[13,14]$. Intuitively, in those instances, OP would decrease the risk of recurrent abscess formation and small bowel fistula that can potentially occur when the small bowel descend into such an irradiated abscess cavity. The indication for OP therefore might be reserved for the treatment of pelvic sepsis originating from secondary complications and inflammatory bowel disease, rather than primary APR for cancer, although there is no clear evidence to support this.

In fact, in this study we found a higher incidence of wound breakdown and delayed healing in those patients with an insufficient OP. Several explanations can be considered for the poor outcomes in this group. First, the observed poor healing rates may be caused by undetected necrosis of the OP. Most forms of fat necrosis are self-limiting and slowly resolve with conservative management. One year after surgery there is likely to be complete resolution of the necrosis, which may then present as an OP of insufficient length on CT 1 year postoperatively. A second reason may be related to residual dead space formation underneath the OP, caused by inadequate mobilisation of the omental pedicle. This residual dead space distal to the OP is likely to be filled with fluid with subsequent contamination and abscess

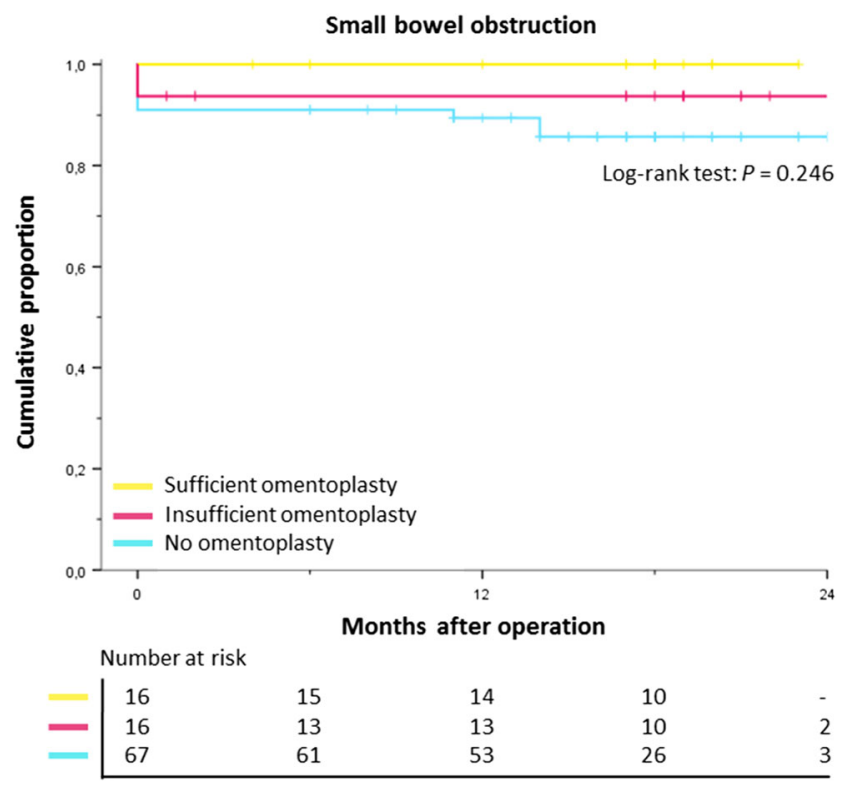

Fig. 4 Kaplan-Meier curve for small bowel obstruction with a pelvic cause after abdominoperineal resection, stratified by the use and quality of omentoplasty formation, resulting in chronic wound problems. However, if insufficient $\mathrm{OP}$ was just related to technical aspects (i.e. no length or poor mobilisation), we would not expect such a large disadvantage compared with no OP.

The higher perineal hernia rate after $\mathrm{OP}$ is in line with previous studies [7, 8]. Although a causal relation cannot be confirmed, the hypothesis is that the pedicled omentum will function as a large amount of loose fatty tissue that will easily descend through the pelvic outlet in the absence of a pelvic floor, while a few loops of small bowel are restricted by a certain mesenteric length in the possibility of herniating at the level of the perineum. The OP is still often perceived as a method for surgical reconstruction of the pelvic floor, but the high rate of omental hernias in those with OP of adequate length and size suggests the opposite. An unexpected but understandable finding was the higher rate of perineal hernias in those with an insufficient OP. Patients in this group showed a significant increase in chronic wound problems. Such chronic wound infections are known to weaken the pelvic scar, likely causing the observed increase in perineal hernia formation. The comparison may also be partly skewed by the higher frequency of hysterectomy in patients with an insufficient OP, although we corrected for this confounder in Cox regression.

Although this study did not find a significant reduction of small bowel obstruction, it was notable that none of the patients with OP of sufficient length and volume experienced small bowel obstruction at the level of the pelvic cavity. It could therefore be that a true effect may not be demonstrated due to relatively low numbers. However, as only few patients would require surgical management, and this study as well as two recent large studies showed no significant reduction in reoperation rate for ileus $[7,8]$, it is questionable whether this potential effect of OP would be clinically relevant.

There are some uncertainties to the generalisation of these findings, mostly related to the retrospective study design, allowing the potential risk of confounding by indication. Patients selected for OP could potentially have had wider resections that would put them at increased risk for complications, including hernia. Although this is likely to be true for some patients, most patients underwent the procedure that was considered routine care at a certain point in time by a certain operating surgeon. Unfortunately, there was restricted information available in the patient files on reasons for applying OP. Also the surgeon impression of the quality of OP and degree of obliteration was not available in many cases, hampering a comparison between the surgeon impression at the time of operation and CT assessment. Another limitation of this study may be related to the relatively small sample size, and the resulting limited number of covariates that we could include in the multivariable models. Finally, extended resections that required a muscle flap were excluded in the current study, and conclusions on the role of OP cannot necessarily be extrapolated to this subgroup.

To date, there remains no proven benefit of OP for patients that undergo APR for rectal cancer. This study suggests that 
performing OP, even with sufficient length and volume, does not seem to affect perineal wound healing. If the $\mathrm{OP}$ appears to be of poor quality on postoperative imaging - due to for example partial necrosis - the outcomes appear to be even worse than not performing an OP. Furthermore, the protective effect in preventing small bowel obstruction remains speculative, and there is likely an elevated risk of developing a perineal hernia after OP if considering recent literature [7-9].

\section{Conclusion}

In this study, OP did not affect perineal wound healing and related morbidity - even in case of adequate length and bulk to fill the pelvic dead space - and insufficient OP was associated with a higher risk of chronic wound problems after APR. These findings are consistent with recent literature and suggest that $\mathrm{OP}$ is not indicated for primary filling of the empty space after APR for rectal cancer. But, considering the limitations pertaining to a relatively small retrospective study, future studies are necessary to validate the proposed method of postoperative $\mathrm{CT}$ assessment of the quality of an omentoplasty and to confirm our findings.

Acknowledgement The authors would like to thank Daniel A.J. Murphy for his assistance in the data acquisition. The study was sponsored by the Oxford University Hospitals NHS Foundation Trust.

\section{Compliance with ethical standards}

The study received approval of the local Institutional Review Board (Cambridge East Research Ethics Committee). Patients were studied as part of a retrospective surgical audit and written informed consent was not required.

Conflict of interest The authors declare that they have no conflict of interest.

Open Access This article is distributed under the terms of the Creative Commons Attribution 4.0 International License (http:// creativecommons.org/licenses/by/4.0/), which permits unrestricted use, distribution, and reproduction in any medium, provided you give appropriate credit to the original author(s) and the source, provide a link to the Creative Commons license, and indicate if changes were made.

\section{References}

1. Musters GD, Klaver CEL, Bosker RJI, Burger JWA, van Duijvendijk P, van Etten B, van Geloven AAW, de Graaf EJR, Hoff C, Leijtens JWA, Rutten HJT, Singh B, Vuylsteke R, de Wilt JHW, Dijkgraaf MGW, Bemelman WA, Tanis PJ (2017) Biological mesh closure of the pelvic floor after extralevator abdominoperineal resection for rectal cancer: a multicenter randomized controlled trial (the BIOPEX-study). Ann Surg 265(6): 1074-1081. https://doi.org/10.1097/SLA.0000000000002020

2. Asplund D, Prytz M, Bock D, Haglind E, Angenete E (2015) Persistent perineal morbidity is common following abdominoperineal excision for rectal cancer. Int $\mathrm{J}$ Color Dis 30(11):1563-1570. https://doi.org/10.1007/s00384-015-2328-1

3. Imaizumi K, Nishizawa Y, Ikeda K, Tsukada Y, Sasaki T, Ito M (2018) Extended pelvic resection for rectal and anal canal tumors is a significant risk factor for perineal wound infection: a retrospective cohort study. Surg Today 48(11):978-985. https://doi.org/10.1007/ s00595-018-1680-5

4. Musters GD, Buskens CJ, Bemelman WA, Tanis PJ (2014) Perineal wound healing after abdominoperineal resection for rectal cancer: a systematic review and meta-analysis. Dis Colon Rectum 57(9): 1129-1139. https://doi.org/10.1097/DCR.0000000000000182

5. West NP, Anderin C, Smith KJ, Holm T, Quirke P, European Extralevator Abdominoperineal Excision Study G (2010) Multicentre experience with extralevator abdominoperineal excision for low rectal cancer. Br J Surg 97(4):588-599. https://doi. org/10.1002/bjs.6916

6. Hultman CS, Sherrill MA, Halvorson EG, Lee CN, Boggess JF, Meyers MO, Calvo BA, Kim HJ (2010) Utility of the omentum in pelvic floor reconstruction following resection of anorectal malignancy: patient selection, technical caveats, and clinical outcomes. Ann Plast Surg 64(5):559-562. https://doi.org/10.1097/SAP. 0b013e3181ce3947

7. Blok RD, de Jonge J, de Koning MA, van de Ven AWH, van der Bilt JDW, van Geloven AAW, Hompes R, Bemelman WA, Tanis PJ (2019) Propensity score adjusted comparison of pelviperineal morbidity with and without omentoplasty following abdominoperineal resection for primary rectal cancer. Dis Colon Rectum. https://doi. org/10.1097/DCR.0000000000001349

8. Blok RD, Musters GD, WAA B, Buskens CJ, Bemelman WA, Tanis PJ, Collaborative Dutch Snapshot Research G (2018) Snapshot study on the value of omentoplasty in abdominoperineal resection with primary perineal closure for rectal cancer. Ann Surg Oncol 25(3):729-736. https://doi.org/10.1245/s10434-017-6273-9

9. Blok RD, Hagemans JAW, Klaver CEL, Hellinga J, van Etten B, Burger JWA, Verhoef C, Hompes R, Bemelman WA, Tanis PJ (2019) A systematic review and meta-analysis on omentoplasty for the management of abdominoperineal defects in patients treated for cancer. Ann Surg. https://doi.org/10.1097/SLA.0000000000003266

10. Munkedal DLE, Rosenkilde M, Nielsen DT, Sommer T, West NP, Laurberg S (2017) Radiological and pathological evaluation of the level of arterial division after colon cancer surgery. Color Dis 19(7): O238-O245. https://doi.org/10.1111/codi.13756

11. von Elm E, Altman DG, Egger M, Pocock SJ, Gotzsche PC, Vandenbroucke JP, Initiative S (2008) The Strengthening the Reporting of Observational Studies in Epidemiology (STROBE) statement: guidelines for reporting observational studies. J Clin Epidemiol 61(4):344-349. https://doi.org/10.1016/j.jclinepi.2007. 11.008

12. Musters GD, Lapid O, Stoker J, Musters BF, Bemelman WA, Tanis PJ (2016) Is there a place for a biological mesh in perineal hernia repair? Hernia 20(5):747-754. https://doi.org/10.1007/s10029-0161504-8

13. Sloothaak DA, Buskens CJ, Bemelman WA, Tanis PJ (2013) Treatment of chronic presacral sinus after low anterior resection. Color Dis 15(6):727-732. https://doi.org/10.1111/codi.12094

14. Musters GD, Borstlap WA, Bemelman WA, Buskens CJ, Tanis PJ (2016) Intersphincteric completion proctectomy with omentoplasty for chronic presacral sinus after low anterior resection for rectal cancer. Color Dis 18(2):147-154. https://doi.org/10.1111/codi. 13086

Publisher's note Springer Nature remains neutral with regard to jurisdictional claims in published maps and institutional affiliations. 